\title{
Reproducing kernel approach for numerical solutions of fuzzy fractional initial value problems under the Mittag-Leffler kernel differential operator
}

\author{
omar abu arqub ${ }^{1}$, Jagdev Singh ${ }^{2}$, Banan Maayah $^{3}$, and Mohammed Alhodaly ${ }^{4}$
}

${ }^{1}$ Al-Balqa' Applied University

${ }^{2}$ JECRC University

${ }^{3}$ The University of Jordan

${ }^{4}$ King Abdulaziz University

January 5, 2021

\begin{abstract}
In this research study, fuzzy fractional differential equations in presence of the Atangana-Baleanu-Caputo differential operators are analytically and numerically treated using extended reproducing Kernel Hilbert space technique. With the utilization of a fuzzy strongly generalized differentiability form, a new fuzzy characterization theorem beside two fuzzy fractional solutions is constructed and computed. To besetment the attitude of fuzzy fractional numerical solutions; analysis of convergence and conduct of error beyond the reproducing kernel theory are explored and debated. In this tendency, three computational algorithms and modern trends in terms of analytic and numerical solutions are propagated. Meanwhile, the dynamical characteristics and mechanical features of these fuzzy fractional solutions are demonstrated and studied during two applications via three-dimensional graphs and tabulated numerical values. In the end, highlights and future suggested research work are eluded.
\end{abstract}

\section{Hosted file}

The Paper.pdf available at https://authorea.com/users/370043/articles/502479-reproducingkernel-approach-for-numerical-solutions-of-fuzzy-fractional-initial-value-problemsunder-the-mittag-leffler-kernel-differential-operator 